\title{
Editorial
}

\section{Models of healthcare in respiratory diseases}

Increasing healthcare cost and utilisation of health services have sparked an interest in new models of care, especially those that allow provision of traditional inpatient care in a community setting. COVID-19 has accelerated the implementation of telehealth, the delivery of medical care via phone or digital communication.

Remote monitoring of patients with COVID-19 who are well enough to stay at home but are at risk of clinical deterioration allows us to monitor changes in symptoms and vital signs such as oxygen saturation $\left(\mathrm{SpO}_{2}\right)$, heart rate, blood pressure and temperature. Monitoring is enabled by surveys/ diaries [1] and connected devices [2]. This facilitates timely intervention by the care team if the patient's condition worsens. Remote monitoring of $\mathrm{SpO}_{2}$ has also been used in patients with COVID-19 pneumonia discharged from hospital to detect those who acutely deteriorate and require urgent assessment and potential hospital readmission [2]. Monitoring at home has the potential to reduce the need for hospital admissions and the length of hospital stays, therefore allowing focusing of resources on those most in need of hospital care.

Chronic respiratory diseases may lend themselves to remote monitoring. With the current push to digital health, we will probably see an explosion in home monitoring health services. The evidence for the effectiveness of such interventions in chronic respiratory diseases is currently, however, insufficient. Large high-quality randomised trials are required to establish the effectiveness of home monitoring programmes in chronic respiratory diseases before they are rolled out as part of routine healthcare.

In asthma, remote monitoring has been shown to be an effective tool to identify nonadherence in individuals with difficult-to-control asthma using suppression of fractional exhaled nitric oxide with directly observed therapy of inhaled corticosteroids over 7 days [3]. This approach has the potential to identify patients who are likely to respond to highdose inhaled corticosteroids and those who, despite good adherence to inhaled corticosteroids, are likely to require additional treatment [3]. A randomised trial of a home monitoring programme on top of standard care for 24 weeks in patients with idiopathic pulmonary fibrosis did not show any significant difference in the primary outcome of health-related quality of life compared with standard care only [4]. The intervention included home spirometry, reporting of symptoms and sideeffects, patient-reported outcomes, information, a medication coach, and online consultations [4]. In COPD, a three-arm randomised trial evaluating the effectiveness of a remote monitoring and a selfmonitoring programme did not show any significant differences in self-management skills, knowledge, symptoms, or healthcare use compared with each other or with a standard care group [5].

COVID-19 has highlighted a lack of care coordination between hospitals and aged care homes, a problem that is often encountered in patients with chronic conditions as well.

In this issue of Breathe, different aspects of health service provision to patients with

@ERSpublications

The September issue of Breathe focuses on models of healthcare in respiratory diseases: read the introductory editorial by Chief Editor @ClaudiaCDobler https://bit.ly/2YTcl8V

Cite as: Dobler CC. Models of healthcare in respiratory diseases. Breathe 2020; 16: 200213.

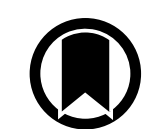

CrossMark 
respiratory diseases will be highlighted, including improving continuity of care of respiratory patients at time of hospital admission and discharge [6], multidisciplinary care for patients with neuromuscular disease [7], outpatient treatment for pulmonary embolism, and the timing of referring patients with COPD to outpatient palliative care [8].

\section{Affiliations}

Claudia C. Dobler ${ }^{1,2}$

${ }^{1}$ Institute for Evidence-Based Healthcare, Bond University, Robina, Australia. ${ }^{2}$ Dept of Respiratory Medicine, Liverpool Hospital, Sydney, Australia.

\section{Conflict of interest}

None declared.

\section{References}

1. Annis T, Pleasants S, Hultman G, et al. Rapid implementation of a COVID-19 remote patient monitoring program. J Am Med Inform Assoc 2020; 27: 1326-1330.

2. O'Carroll O, MacCann R, O'Reilly A, et al. Remote monitoring of oxygen saturation in individuals with COVID-19 pneumonia. Eur Respir J 2020; 56: 2001492

3. Heaney LG, Busby J, Bradding P, et al. Remotely monitored therapy and nitric oxide suppression identifies nonadherence in severe asthma. Am J Respir Crit Care Med 2019; 199: 454-464.

4. Moor CC, Mostard RLM, Grutters JC, et al. Home monitoring in patients with idiopathic pulmonary fibrosis. a randomized controlled trial. Am J Respir Crit Care Med 2020; 202: 393-401.
5. Stamenova V, Liang K, Yang R, et al. Technology-enabled selfmanagement of chronic obstructive pulmonary disease with or without asynchronous remote monitoring: randomized controlled trial. J Med Internet Res 2020; 22: e18598.

6. Dummer J, Stokes T. Improving continuity of care of patients with respiratory disease at hospital discharge. Breathe 2020; 16: 200161.

7. Shah NM, Murphy PB, Kaltsakas G. The adult multidisciplinary respiratory neuromuscular clinic. Breathe 2020; 16: 200121.

8. Strutt R. When to refer patients with advanced COPD to palliative care services. Breathe 2020; 16: 200061. 